\title{
COMPUTATION OF THE TEST STATISTIC AND THE NULL DISTRIBUTION IN THE MULTIVARIATE ANALOGUE OF THE ONE-SIDED TEST
}

\author{
Yoshiro Yamamoto*, Akio Kudồ and Katsumi Ujiie ${ }^{\ddagger}$
}

\begin{abstract}
The multivariate analogue of the one sided test derived in Kudô (1963) is considered. A handy method of computing the test statistic and its significance probability is given. The method is based on applying sweep out operations on a certain matrix in a systematic manner and applying the Fortran subroutine of Sun (1988).
\end{abstract}

\section{INTRODUCTION}

The problem of this paper is as follows. Given a $p$-variate normal distribution: $N_{p}(\boldsymbol{\theta}, \boldsymbol{\Lambda})$, we consider the testing problem: $\mathrm{H}_{0}: \boldsymbol{\theta}=\mathbf{0}$ versus $\mathrm{H}_{1}-\mathrm{H}_{0}: \boldsymbol{\theta} \geq \mathbf{0}$, where $\boldsymbol{\Lambda}$ is assumed to be known and $\boldsymbol{\theta}=\left(\theta_{1}, \cdots, \theta_{p}\right)^{\prime} \geq 0$ means $\operatorname{Max} \theta_{i}>0$, Min $\theta_{i} \geq 0$, thus the space of the alternative hypothesis is a cone. The MLE of $\boldsymbol{\theta}$ in the space of $\mathrm{H}_{0} \cup \mathrm{H}_{1}$ is often called "the projection into the cone" because of the very nature of the alternative hypothesis. In this paper we also set the hypothesis $\mathrm{H}_{2}: \boldsymbol{\theta} \neq \mathbf{0}$, and consider a testing problem : $\mathrm{H}_{1}$ versus $\mathrm{H}_{2}-\mathrm{H}_{1}$.

More than 3 decades have passed since the paper "Multivariate Analogue of the Onesided Test" was published in Biometrika where the above problem was treated and the likelihood ratio test was derived. This paper has been criticized in two aspects. Difficulties in computing (i) the test statistic and (ii) the significance probability. (See for instance Tang et al. (1989). See also Barlow et al. (1972) and Robertson et al. (1988).)

Our purpose is to demonstrate methods to compute these two. In Section 2 we quickly review the outline of the problem, and Section 3 is devoted to the method for computing the MLE, which will find its application in computing an optimum linear test given in Shi et al. (1987), and Section 4 to a numerical example. In Section 5 an application of Sun's subroutine is demonstrated and its accuracy examined, and the final Section is for concluding discussions.

The first of two distribution functions given in Section 2 was given by Kudô (1963) and the second can be derived from Theorem 2.3.1. of Robertson et al. (1988). The second distribution was treated in Robertson and Wegman (1978) and we documented the same in the present paper's form.

\footnotetext{
* Graduate School of Natural Science and Technology, Okayama University, 3-1-1, Tsushima-naka, Okayama 700-0082, Japan

† Faculty of Economics and Information Science, Hyogo University, 2301, Shinzaike, Hiraoka-Cho, Kakogawa 675-0101, Japan

$\ddagger$ Department of Mathematics, School of Science, Tokai University, 1117, Kitakaname, Hiratsuka 2591292, Japan

Key words: Level probability; Orthant probability; Order restricted statistical inference.
} 


\section{A BRIEF REVIEW}

As the variance matrix $\Lambda$ is assumed to be known, we can assume there is only one observation $x$. Let $P$ be the set of integers $P=\{1, \cdots, p\}$ and $M=\left\{i_{1}, \cdots, i_{m}\right\}$ be a subset of $P$. It was shown that there exists uniquely a set satisfying the condition stated below. (see Beale (1959))

Without loss of generality, we state the condition in the case when the set is the first $m$ natural numbers: $M=\{1, \cdots, m\}$ where $m \leq p$.

Let the observation vector $\boldsymbol{x}^{\prime}=\left(x_{1}, \cdots, x_{p}\right)$ be partitioned into $m$ and $p-m$ components and the mean vector and the variance matrix be partitioned accordingly:

$$
\boldsymbol{x}=\left(\begin{array}{l}
\boldsymbol{x}_{(1)} \\
\boldsymbol{x}_{(2)}
\end{array}\right), \boldsymbol{\theta}=\left(\begin{array}{l}
\boldsymbol{\theta}_{(1)} \\
\boldsymbol{\theta}_{(2)}
\end{array}\right), \quad \boldsymbol{\Lambda}=\left(\begin{array}{ll}
\boldsymbol{\Lambda}_{11} & \boldsymbol{\Lambda}_{12} \\
\boldsymbol{\Lambda}_{21} & \boldsymbol{\Lambda}_{22}
\end{array}\right) .
$$

There exists uniquely the subset $M$ satisfying the condition.

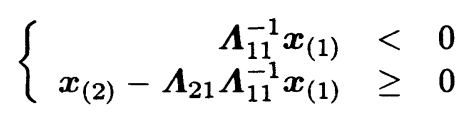

When $m=0, M=\phi$, the empty set, and when $m=p$, the second condition in (1) is vacuous.

The MLE under $H_{0} \cup H_{1}$ is given by

$$
\hat{\boldsymbol{\theta}}=\left(\begin{array}{c}
\hat{\boldsymbol{\theta}}_{(1)} \\
\hat{\boldsymbol{\theta}}_{(2)}
\end{array}\right)=\left(\begin{array}{c}
\mathbf{0} \\
\boldsymbol{x}_{(2)}-\boldsymbol{\Lambda}_{21} \boldsymbol{\Lambda}_{11}^{-1} \boldsymbol{x}_{(1)}
\end{array}\right)
$$

and the test statistic $\bar{\chi}^{2}$ for the testing problem $\mathrm{H}_{0}$ versus $\mathrm{H}_{1}-\mathrm{H}_{0}$ and the one $\overline{\bar{\chi}}^{2}$ for $\mathrm{H}_{1}$ versus $\mathrm{H}_{2}-\mathrm{H}_{1}$ are respectively given by the following form.

$$
\begin{aligned}
\bar{\chi}^{2} & \left.=-2 \log \text { (likelihood ratio of } \mathrm{H}_{0} \text { versus } \mathrm{H}_{1}-\mathrm{H}_{0}\right) \\
& =\boldsymbol{x}^{\prime} \boldsymbol{\Lambda}^{-1} \boldsymbol{x}-(\boldsymbol{x}-\hat{\boldsymbol{\theta}})^{\prime} \boldsymbol{\Lambda}^{-1}(\boldsymbol{x}-\hat{\boldsymbol{\theta}}) \\
& =\hat{\boldsymbol{\theta}}^{\prime} \boldsymbol{\Lambda}^{-1} \hat{\boldsymbol{\theta}} \\
& =\boldsymbol{x}^{\prime} \boldsymbol{\Lambda}^{-1} \boldsymbol{x}-\boldsymbol{x}_{(1)}^{\prime} \boldsymbol{\Lambda}_{11}^{-1} \boldsymbol{x}_{(1)} \\
\overline{\bar{\chi}}^{2} & =-2 \log \left(\text { likelihood ratio of } \mathrm{H}_{1} \text { versus } \mathrm{H}_{2}-\mathrm{H}_{1}\right) \\
& =(\boldsymbol{x}-\hat{\boldsymbol{\theta}})^{\prime} \boldsymbol{\Lambda}^{-1}(\boldsymbol{x}-\hat{\boldsymbol{\theta}}) \\
& =\boldsymbol{x}_{(1)}^{\prime} \boldsymbol{\Lambda}_{11}^{-1} \boldsymbol{x}_{(1)} .
\end{aligned}
$$

The test statistics $\bar{\chi}^{2}$ and $\overline{\bar{\chi}}^{2}$ are, under $\mathrm{H}_{0}$, distributed in the mixtures of the $\chi^{2}$ distributions. For positive constants, $\bar{\chi}_{0}^{2}$ and $\overline{\bar{\chi}}_{0}^{2}$, we have the following identity.

$$
\begin{aligned}
& \operatorname{pr}\left\{\bar{\chi}^{2} \geq \bar{\chi}_{0}^{2}\right\}=\sum_{k=0}^{p} W(k, p) \operatorname{pr}\left\{\chi_{k}^{2} \geq \bar{\chi}_{0}^{2}\right\}, \\
& \operatorname{pr}\left\{\overline{\bar{\chi}}^{2} \geq \overline{\bar{\chi}}_{0}^{2}\right\}=\sum_{k=0}^{p} W(k, p) \operatorname{pr}\left\{\chi_{p-k}^{2} \geq \overline{\bar{\chi}}_{0}^{2}\right\},
\end{aligned}
$$

where $\chi_{k}^{2}$ is a random variable distributed in $\chi^{2}$ distribution with the degrees of freedom $k$ and when $k=0, \operatorname{pr}\left\{\chi_{0}^{2}=0\right\}=1$ is assumed and $W(k, p)$ is a constant given below. Parallel 
relations are given by Theorem 2.3.1 in Robertson et al. (1988), where mixtures of Beta distributions are also given. This will be discussed in the final section.

The constants, $W(k, p)$, or the weights in the mixtures, are the sums of the products of two orthant probabilities. The orthant probability of a positive definite matrix $\boldsymbol{\Sigma}$, denoted by $Q\{\boldsymbol{\Sigma}\}$, is the probability that the random vector distributed in $N(\mathbf{0}, \boldsymbol{\Sigma})$ has components all positive.

The constants are sometimes called level probability, in order to avoid confusion with the terminology with "weights" in isotonic regression, and are given by

$$
W(k, p)=\sum_{M: n(M)=k} Q\left\{\boldsymbol{\Lambda}_{M: M^{c}}\right\} Q\left\{\left(\boldsymbol{\Lambda}_{M^{c}}\right)^{-1}\right\}
$$

where the summation is of ${ }_{p} C_{k}$ terms for all subsets of $P$ of size $k$ or equivalently, $n(M)=k$, where $n(M)$ is the number of elements in $M, \boldsymbol{\Lambda}_{M: M^{c}}$ is the $m$-dimensional variance matrix of $x_{i}$ for $i \in M$ under the condition $x_{i}=0$ for $i \notin M$, and $\left(\boldsymbol{\Lambda}_{M^{c}}\right)^{-1}$ is the inverse of the $(p-k)$ dimensional variance matrix of $x_{i}$ for $i \notin M$.

\section{A METHOD FOR COMPUTING MLE AND THE VALUE OF THE TEST STATISTICS}

In order to demonstrate how to compute MLE, we need to give some considerations to sweep out operations. Consider the following $(p+1) \times(p+1)$ matrix:

$$
\left(\begin{array}{ccc}
\boldsymbol{\Lambda}_{11} & \boldsymbol{\Lambda}_{12} & \boldsymbol{x}_{(1)} \\
\boldsymbol{\Lambda}_{21} & \boldsymbol{\Lambda}_{22} & \boldsymbol{x}_{(2)} \\
\boldsymbol{x}_{(1)}^{\prime} & \boldsymbol{x}_{(2)}^{\prime} & 0
\end{array}\right)
$$

Application of $m(<p)$ times of successive sweep out operations in the ordinary way makes the upper left square portion the $m \times m$ unit matrix. The right hand side in (4) is the result of these operations and the first factor in the left hand side is the matrix representing them.

$$
\begin{aligned}
& \left(\begin{array}{ccc}
\boldsymbol{\Lambda}_{11}^{-1} & \mathbf{O} & \mathbf{0} \\
-\boldsymbol{\Lambda}_{21} \boldsymbol{\Lambda}_{11}^{-1} & \mathbf{E}_{2} & \mathbf{0} \\
-\boldsymbol{x}_{(1)}^{\prime} \boldsymbol{\Lambda}_{11}^{-1} & \mathbf{0}^{\prime} & 1
\end{array}\right)\left(\begin{array}{ccc}
\boldsymbol{\Lambda}_{11} & \boldsymbol{\Lambda}_{12} & \boldsymbol{x}_{(1)} \\
\boldsymbol{\Lambda}_{21} & \boldsymbol{\Lambda}_{22} & \boldsymbol{x}_{(2)} \\
\boldsymbol{x}_{(1)}^{\prime} & \boldsymbol{x}_{(2)}^{\prime} & 0
\end{array}\right) \\
& =\left(\begin{array}{ccc}
\mathbf{E}_{1} & \boldsymbol{\Lambda}_{11}^{-1} \boldsymbol{\Lambda}_{12} & \boldsymbol{\Lambda}_{11}^{-1} \boldsymbol{x}_{(1)} \\
\mathbf{O} & \boldsymbol{\Lambda}_{22}-\boldsymbol{\Lambda}_{21} \boldsymbol{\Lambda}_{11}^{-1} \boldsymbol{\Lambda}_{12} & \boldsymbol{x}_{(2)}-\boldsymbol{\Lambda}_{21} \boldsymbol{\Lambda}_{11}^{-1} \boldsymbol{x}_{(1)} \\
\mathbf{0}^{\prime} & \boldsymbol{x}_{(2)}^{\prime}-\boldsymbol{x}_{(1)}^{\prime} \boldsymbol{\Lambda}_{11}^{-1} \boldsymbol{\Lambda}_{12} & -\boldsymbol{x}_{(1)}^{\prime} \boldsymbol{\Lambda}_{11}^{-1} \boldsymbol{x}_{(1)}
\end{array}\right)
\end{aligned}
$$

We call $\boldsymbol{\Lambda}_{11}$ the pivotal matrix. The right hand side is the result of the sweeping out with $\boldsymbol{\Lambda}_{11}$ as the pivot, and to arrive at this result there are as many as $m$ ! different paths to it, namely the result is independent of in what order diagonal elements are used as the pivotal element. This fact is important in devising our method.

Now we describe how the test statistic can be found out. First generate an increasing sequence of binary numbers as shown in Table 1. Next reserve spaces in a computer to accommodate $p+1(p+1) \times(2 p+1)$-matrices $S_{0}, \cdots, S_{p}$. And put

$$
\left(\begin{array}{ccccc}
\boldsymbol{\Lambda}_{11} & \boldsymbol{\Lambda}_{12} & \boldsymbol{x}_{(1)} & \mathbf{E}_{1} & \mathrm{O} \\
\boldsymbol{\Lambda}_{21} & \boldsymbol{\Lambda}_{22} & \boldsymbol{x}_{(2)} & \mathrm{O} & \mathbf{E}_{2} \\
\boldsymbol{x}_{(1)}^{\prime} & \boldsymbol{x}_{(2)}^{\prime} & 0 & \mathbf{0}^{\prime} & \mathbf{0}^{\prime}
\end{array}\right)
$$


Table 1. The first $2^{p}-1$ binary numbers.

\begin{tabular}{rrc}
\hline No. & Binary No. & Sweep out position \\
\hline 1 & 1 & $1000 \cdots 0$ \\
2 & 10 & $0100 \cdots 0$ \\
3 & 11 & $1100 \cdots 0$ \\
4 & 100 & $0010 \cdots 0$ \\
$\vdots$ & $\vdots$ & $\vdots$ \\
$2^{p-2}$ & $1 \cdots 1$ & $111 \cdots 10$ \\
$2^{p-2}+1$ & $10 \cdots 0$ & $000 \cdots 01$ \\
$\vdots$ & $\vdots$ & $\vdots$ \\
$2^{p-1}$ & $11 \cdots 1$ & $111 \cdots 11$ \\
\hline
\end{tabular}

The numbers represent the subsets of $P=\{1, \cdots p\}$. For instance the first and the second number represents the subsets consisting of 1 and 2 only, and the third the subsets $\{1,2\}$, and so on. Finally the last one represents the total set $P$.

into $S_{0}$.

Here we start a sequence of sweep out operations, given as follows. First look at the first row of the sequence of binary numbers. Only the last digit on the first row is 1 . This directs us to sweep out the matrix in $S_{0}$ making its $(1,1)$ element as the pivot and put the outcome into $S_{1}$.

Similarly look at the second row, and sweep out the matrix in $S_{0}$ making its $(2,2)$ element as the pivot and put the outcome into $S_{1}$. Then we come to the third row. The only difference lies in the last digit, which is 1 instead of 0 . Thus we sweep out the matrix in $S_{1}$ making its $(1,1)$ element as the pivot and put the outcome into $S_{2}$.

We proceed in this manner until we arrive at the last row, and when we complete the operations corresponding to the last row we will have completed all possible types of sweep out operations. If we have space for keeping records of a certain parts of the outcomes we are ready to compute the significance probability. A numerical example is demonstrated in the next section.

If we look into the intermediate steps, corresponding to (4), it is the matrix of the form:

$$
\left(\begin{array}{ccccc}
\mathbf{E}_{1} & \boldsymbol{\Lambda}_{11}^{-1} \boldsymbol{\Lambda}_{12} & \boldsymbol{\Lambda}_{11}^{-1} \boldsymbol{x}_{(1)} & \boldsymbol{\Lambda}_{11}^{-1} & \mathbf{O} \\
\mathbf{O} & \boldsymbol{\Lambda}_{22: 1} & \boldsymbol{x}_{(2)}-\boldsymbol{\Lambda}_{21} \boldsymbol{\Lambda}_{11}^{-1} x_{(1)} & -\boldsymbol{\Lambda}_{21} \boldsymbol{\Lambda}_{11}^{-1} & \mathbf{E}_{2} \\
\mathbf{0}^{\prime} & \boldsymbol{x}_{(2)}^{\prime}-\boldsymbol{x}_{(1)}^{\prime} \boldsymbol{\Lambda}_{11}^{-1} \boldsymbol{\Lambda}_{12} & -\boldsymbol{x}_{(1)}^{\prime} \boldsymbol{\Lambda}_{11}^{-1} \boldsymbol{x}_{(1)} & -\boldsymbol{x}_{(1)}^{\prime} \boldsymbol{\Lambda}_{11}^{-1} & \mathbf{0}^{\prime}
\end{array}\right)
$$

The condition (1) can be easily checked by looking at the matrix. When the condition is satisfied the MLE is given by the rule

$$
\left\{\begin{array} { r } 
{ \boldsymbol { \Lambda } _ { 1 1 } ^ { - 1 } \boldsymbol { x } _ { ( 1 ) } < 0 } \\
{ \boldsymbol { x } _ { ( 2 ) } - \boldsymbol { \Lambda } _ { 2 1 } \boldsymbol { \Lambda } _ { 1 1 } ^ { - 1 } \boldsymbol { x } _ { ( 1 ) } \geq 0 }
\end{array} \Longrightarrow \left\{\begin{array}{l}
\hat{\boldsymbol{\theta}}_{(1)}=\mathbf{0} \\
\hat{\boldsymbol{\theta}}_{(2)}=\boldsymbol{x}_{(2)}-\boldsymbol{\Lambda}_{21} \boldsymbol{\Lambda}_{11}^{-1} \boldsymbol{x}_{(1)} .
\end{array}\right.\right.
$$

The value of $\overline{\bar{\chi}}^{2}=\boldsymbol{x}_{(1)}^{\prime} \boldsymbol{\Lambda}_{11}^{-1} \boldsymbol{x}_{(1)}$ can be found in the middle of the bottom row in (5) 
At the final step, $m=p$ and, in order to express the outcome, $\boldsymbol{\Lambda}$ and $\boldsymbol{x}$ need not be partitioned and we have

$$
\left(\begin{array}{ccc}
\mathbf{E}_{1} & \boldsymbol{\Lambda}^{-1} \boldsymbol{x} & \boldsymbol{\Lambda}-1 \\
\mathbf{0}^{\prime} & -\boldsymbol{x}^{\prime} \boldsymbol{\Lambda}^{-1} \boldsymbol{x} & -\boldsymbol{x}^{\prime} \boldsymbol{\Lambda}^{-1}
\end{array}\right) .
$$

In the middle of the bottom row in (6), we have the negative value of the ordinary $\chi^{2}=\boldsymbol{x}^{\prime} \boldsymbol{\Lambda}^{-1} \boldsymbol{x}$ and the value of $\bar{\chi}^{2}$ can be obtained by subtraction.

$$
\bar{\chi}^{2}=\chi^{2}-\overline{\bar{\chi}}^{2}
$$

\section{NUMERICAL EXAMPLES}

In this section we present the correction of the example given in Kudô (1963). For this purpose we introduce new notations as follows.

$$
\begin{aligned}
\boldsymbol{x}_{M} & =\left(\begin{array}{c}
\boldsymbol{\Lambda}_{11}^{-1} \boldsymbol{x}_{(1)} \\
\boldsymbol{x}_{(2)}-\boldsymbol{\Lambda}_{21} \boldsymbol{\Lambda}_{11}^{-1} \boldsymbol{x}_{(1)}
\end{array}\right), \\
\overline{\bar{\chi}}_{M}^{2} & =\boldsymbol{x}_{(1)}^{\prime} \boldsymbol{\Lambda}_{11}^{-1} \boldsymbol{x}_{(1)} .
\end{aligned}
$$

The notations in the left hand sides are new and those in the right hand sides are currently used ones. The notation $M$ is used intentionally to emphasize that subset $M$ varies all possible subsets.

\subsection{The first numerical example in Kudô (1963)}

In his paper an example is given as the mean of ten observations. The observed mean vector : $\boldsymbol{x}^{\prime}=\left(x_{1}, x_{2}, x_{3}, x_{4}\right)$ and its variance matrix : $\boldsymbol{\Sigma}$ are the following.

$$
\begin{aligned}
\boldsymbol{x}^{\prime} & \left(\begin{array}{rrrr}
-10.0 & -1.00 & 10.0 & 0.30
\end{array}\right) \\
\boldsymbol{\Sigma} & =\frac{1}{10}\left(\begin{array}{rrrr}
1.00 & 0.20 & 0.20 & -0.10 \\
0.20 & 1.04 & 0.24 & -0.42 \\
0.20 & 0.24 & 1.08 & -0.20 \\
-0.10 & -0.42 & -0.20 & 1.18
\end{array}\right)
\end{aligned}
$$

We need $2^{4}-1=15$ sweep out operations, and their result is given in Table 2 .

In the tenth row in Table 2 , the set $M$ coincides with $N$, indicating that the MLE is given by this sweep out operation. The figures in Table 2 leads us to the conclusions.

$$
\begin{aligned}
\hat{\boldsymbol{\theta}}^{\prime} & =(0,0.76,11.89,0) \\
\bar{\chi}_{0}^{2} & =2410.144-1004.188=1405.956 \\
\overline{\bar{\chi}}_{0}^{2} & =1004.188
\end{aligned}
$$

Although the estimate $\hat{\boldsymbol{\theta}}$, in Kudô (1963), was correct, the value of $\bar{\chi}_{0}^{2}$ was in error and the value of $\overline{\bar{\chi}}_{0}^{2}$ was presented.

\subsection{The second numerical example in Kudô (1963)}

His second example is taken from the study on four anthropometric measurements, where the estimated regression vector is and its estimated variance are given by

$$
\hat{\boldsymbol{b}}^{\prime}=(2.710078,0.774578,0.067798,0.329031)
$$


Table 2. Results of all possible sweep out operations

\begin{tabular}{|c|c|c|c|c|c|c|}
\hline$M$ & \multicolumn{4}{|c|}{$\boldsymbol{x}_{M}$} & $N$ & $\overline{\bar{\chi}}_{M}^{2}$ \\
\hline 0000 & -10.00 & 1.00 & 10.00 & 0.30 & 1000 & 0.0000 \\
\hline 1000 & -10.00 & 1.00 & 12.00 & -0.70 & 1001 & 1000.0000 \\
\hline 0100 & -9.81 & -0.96 & 10.23 & -0.10 & 1101 & 9.6154 \\
\hline 1100 & -10.20 & 1.00 & 11.80 & -0.30 & 1001 & 1010.0000 \\
\hline 0010 & -11.85 & -3.22 & 9.26 & 2.15 & 1100 & 925.9259 \\
\hline 1010 & -12.31 & -1.31 & 11.54 & 1.38 & 1100 & 2384.6154 \\
\hline 0110 & -11.34 & -3.27 & 9.98 & 0.93 & 1100 & 1031.1562 \\
\hline 1110 & -12.09 & -1.36 & 11.80 & 0.88 & 1100 & 2402.4000 \\
\hline 0001 & -9.97 & -0.89 & 10.05 & 0.25 & 1100 & 0.7627 \\
\hline 1001 & -10.06 & 0.76 & 11.89 & -0.60 & 1001 & 1004.1880 \\
\hline 0101 & -9.81 & -1.00 & 10.22 & -0.10 & 1101 & 09.7221 \\
\hline 1101 & -10.21 & 0.88 & 11.77 & -0.30 & 1001 & 1010.8911 \\
\hline 0011 & -11.73 & -2.52 & 9.61 & 1.88 & 1100 & 966.4388 \\
\hline 1011 & -12.23 & -0.87 & 11.75 & 1.21 & 1100 & 2401.2631 \\
\hline 0111 & -11.34 & -2.91 & 10.08 & 0.93 & 1100 & 1039.7192 \\
\hline 1111 & -12.08 & -1.03 & 11.89 & 0.88 & 1100 & 2410.1440 \\
\hline
\end{tabular}

$M$ is the binary expression of the subset used in computing $x_{M}$ and $\overline{\bar{\chi}}_{M}^{2}$ or the components used as pivots, and $N$ is the components of $x_{M}$ which are negative. In the last column the value of $\overline{\bar{\chi}}_{M}^{2}$ is listed. In the first row the vector $\boldsymbol{x}$ is listed and in the subsequent rows results of the sweep out operations are listed.

$$
\hat{\boldsymbol{\Sigma}}=\frac{0.0001653}{10483}\left(\begin{array}{rrrr}
76,030,317 & 13,725,786 & 5,323,840 & 11,672,377 \\
13,725,786 & 5,389,621 & 1,133,651 & 2,140,074 \\
5,323,840 & 1,133,651 & 1,470,791 & 867,806 \\
11,672,377 & 2,140,074 & 867,806 & 3,372,081
\end{array}\right)
$$

In this case the weights and upper tail probabilities in five terms are given by the following, showing fairly large discrepancies in most of the terms.

$$
\begin{aligned}
& \operatorname{pr}\left\{\bar{\chi}^{2} \geq 9.3078\right\} \\
= & \sum_{k=0}^{4} W(h, 4) \operatorname{pr}\left\{\chi_{k}^{2} \geq 9.3078\right\} \\
= & 0.007180 \times 0+0.07610 \times 0.002282+0.2814 \times 0.009524 \\
& +0.4239 \times 0.02546+0.2114 \times 0.05385 \\
= & 0.02503
\end{aligned}
$$

\section{ACCURACY OF THE COMPUTATION OF ORTHANT PROBABILITY BY SUN'S METHOD}

In the example of the previous section, the solution is arrived at the 10th sweep out operation before completing all possible 16 sweep out operations. 
Table 3. Weights calculated from a variance matrix.

\begin{tabular}{cc}
\hline level & weight \\
\hline$W(0,9)$ & 0.000001231906 \\
$W(1,9)$ & 0.000048507242 \\
$W(2,9)$ & 0.000794223862 \\
$W(3,9)$ & 0.007082204100 \\
$W(4,9)$ & 0.037852858587 \\
$W(5,9)$ & 0.125468639937 \\
$W(6,9)$ & 0.256967597212 \\
$W(7,9)$ & 0.312562319396 \\
$W(8,9)$ & 0.204384091705 \\
$W(9,9)$ & 0.054838330826 \\
\hline
\end{tabular}

By changing the arrangement of components it is possible to arrive at the MLE earlier or later, but all of the steps of sweep out operations are needed for calculating the significance probability.

The first and the second factor in the sum in (2) involve the variance matrices of different dimensions, and they can be found in (5) in the form of $\boldsymbol{\Lambda}_{22: 1}$ and $\boldsymbol{\Lambda}_{11}^{-1}$. About the computation of the orthant probabilities, a powerful Fortran subroutine is available, which can compute the probabilities up to nine dimensions (Sun (1988b)). We have checked its accuracy. In addition to the trivial identity that the sum of the weights should be equal to one, we have another one given by McMullen(1975).

$$
\sum_{k=0}^{p} W(k, p) \times(-1)^{k}=0 .
$$

This was once conjectured by Shapiro (1987). An example was taken from Schull and Neel (1965), which contains tables of the sums of squares and cross products based on some hundreds of observations. This enables us to perform multivariate multiple regression analysis of anthropometric measurements of 10 dimension with 3 independent variables. We selected 9 measurements and performed regression analysis.

Based on this analysis we computed the significance probability of the multivariate analogue of the one sided test. In Table 3 we tabulated the weights. From the table we have

$$
\sum_{i=0}^{9} W(i, 9)=1.000000004772
$$

In addition to this trivial identity, we can check the accuracy by the Shapiro's conjecture.

$$
\sum_{i: \text { odd }} W(i, 9)=0.500000001500 \sum_{i: e v e n} W(i, 9)=0.500000003272
$$

These figures show that the accuracy of Sun's subroutine is very satisfactory. 


\section{CONCLUDING DISCUSSION}

The variance matrix $\Lambda$ was assumed to be known, but this can be relaxed to the case $\Lambda=\sigma^{2} \Lambda_{0}$ with $\Lambda_{0}$ known and $\sigma$ unknown. This is true when we have an estimate of $\sigma$ statistically independent of the observation $x$ distributed in chi-square distribution with some degrees of freedom.

The method described in Section 3. is essentially the same as the one described in Tarumi and Kudô (1974).

The method of computing the projection described in Section 3 can be applied in computing a kind of linear optimal test given in Shi and Kudô (1987) and Shi (1987). This optimum test statistic is nothing but the projection into a cone determined by a variance matrix in a certain way.

In some cases the algorithm for computing MLE is simple and straightforward, and repeated sweep out operations are not needed. Simple order is the typical case and, when weights are equal, the level probabilities can be computed recursively (see Corollary B to Theorem 3.3 in Barlow et al. (1972) and (2.4.11) in Robertson et al. (1988)).

Choi (1976) treated the case when the matrix (3) is positive definite with non-positive off-diagonal elements, which is the matrix version of the case with straightforward algorithm. Even in this case the level probability is not easily computed except for the case of simple order with the same weights. Dr. Ning-Zhong Shi kindly communicated this fact to the authors and indeed mentioned it in Shi (1991), and this amplifies the merit of this paper.

\section{REFERENCES}

Barlow R. E., Bartholomew. D. J., Bremner, J. M. and Brunk, H. D. (1972). Statistical Inference under Order Restrictions, 2nd ed. New York:, John Wiley.

Beale, E. M. L. (1959). On quadratic programming. Naval Research Logistic Quarterly, 6, 227-243.

Choi, J. R. (1976). The law of sweep-out-negatives in the estimation under order restrictions. Memoirs of Faculty of Science, Kyushu Univ., Ser. A, 30, 135-143.

Kudô, A. (1963). A multivariate analogue of one-sided test. Biometrika, 50, 403-18.

McMullen, P. (1975). Non-linear angle-sum relations for polyhedral cones and polytopes. Math. Proc. Camb. Phil. Soc., 78, 247-61.

Robertson, T. and Wegman, E. J. (1978) Likelihood test for order restrictions in exponential families. Ann. Statist., 6, 485-505.

Robertson, T., Wright, F. T. and Dykstra, R. L. (1988). Order Restricted Statistical Inference, New York:, John Wiley.

Schull, W. J. and Neel, J. V. (1965). The Effect of Inbreeding on Japanese Children. Harper \& Row.

Shapiro, A. (1987). A conjecture related to chi-bar-squared distributions. Ameri. math. month., 94, $46-48$.

Shi, N.-Z. (1987). Testing a normal mean vector against the alternative determined by a convex cone. Memoirs of Faculty of Science, Kyushu Univ., Ser. A, 41, 133-145.

Shi, N.-Z.(1991). A test of homogeneity of odds ratios against order restrictions. Journ. American Statist. Assoc. 86, 154-158.

Shi, N.-Z and Kudô, A. (1987). The most stringent somewhere most powerful one sided test of the multivariate normal mean. Memoirs of Faculty of Science, Kyushu Univ., Ser. A, 41, 37-44.

Sun, H.-J. (1988). A Fortran subroutine for computing normal orthant probabilities of dimensions up to nine. Commun. Statist.-Simula., 17, (3), 1097-1111. 
Computation of the Test Statistic and the Null Distribution

Tang, D.-I. Gnecco, C. and Geller, N. L. (1989). An approximate likelihood ratio test for a normal mean vector with nonnegative components with application to clinical trials. Biometrika, $\mathbf{7 6}$, $577-83$.

Tarumi, T. and Kudô, A. (1974). An algorithm related to all possible regression and discriminate analysis. Journ. Japan Statist. Soc., 4, 47-50.

(Received July 1997; Revised December 1997) 